\title{
Placenta-specific protein 1 promotes cell proliferation and invasion in non-small cell lung cancer
}

\author{
LI YANG $^{1}$, TIAN-QI ZHA ${ }^{1}$, XIANG HE ${ }^{2}$, LIANG CHEN ${ }^{3}$, QUAN ZHU ${ }^{3}$, WEI-BING WU ${ }^{3}$, FENG-QI NIE ${ }^{4}$, \\ QIAN WANG ${ }^{1}$, CHONG-SHUANG ZANG $^{1}$, MEI-LING ZHANG $^{1}$, JING HE ${ }^{1}$, \\ WEI LI ${ }^{1}$, WEN JIANG ${ }^{5}$ and KAI-HUA LU ${ }^{1}$ \\ Departments of ${ }^{1}$ Oncology, ${ }^{2}$ Digestive and ${ }^{3}$ Cardiothoracic Surgery, First Affiliated Hospital, \\ ${ }^{4}$ Department of Oncology, Second Affiliated Hospital, and ${ }^{5}$ Department of Biochemistry, \\ Nanjing Medical University, Gulou, Nanjing, Jiangsu 210029, P.R. China
}

Received April 5, 2017; Accepted September 26, 2017

DOI: 10.3892/or.2017.6086

\begin{abstract}
Pulmonary carcinoma-associated proteins have emerged as crucial players in governing fundamental biological processes such as cell proliferation, apoptosis and metastasis in human cancers. Placenta-specific protein 1 (PLAC1) is a cancer-related protein, which is activated and upregulated in a variety of malignant tissues, including prostate cancer, gastric adenocarcinoma, colorectal, epithelial ovarian and breast cancer. However, its biological role and clinical significance in non-small cell lung cancer (NSCLC) development and progression are still unknown. In the present study, we found that PLAC1 was significantly upregulated in NSCLC tissues, and its expression level was associated with advanced pathological stage and it was also correlated with shorter progression-free survival of lung cancer patients. Furthermore, knockdown of PLAC1 expression by siRNA inhibited cell proliferation, induced apoptosis and impaired invasive ability in NSCLC cells partly via regulation of epithelial-mesenchymal transition (EMT)-related protein expression. Our findings present that increased PLAC1 could be identified as a negative prognostic biomarker in NSCLC and regulate cell proliferation and invasion. Thus, we conclusively demonstrated that PLAC1 plays a key role in NSCLC development and progression, which may provide novel insights on the function of tumor-related genedriven tumorigenesis.
\end{abstract}

\section{Introduction}

Non-small cell lung cancer (NSCLC), composed of adenocarcinoma and squamous cell carcinoma, is the preponderant form of lung cancer and accounts for the majority of lung

Correspondence to: Dr Kai-Hua Lu, Department of Oncology, The First Affiliated Hospital of Nanjing Medical University, 300 Guangzhou Road, Gulou, Nanjing, Jiangsu 210029, P.R. China E-mail: 13605179453@126.com; h_1_1726@sina.com

Key words: NSCLC, PLAC1, prognosis biomarker, proliferation, invasion cancer-related deaths worldwide (1-4). Despite advances in the diagnosis and treatment of patients with NSCLC, the overall 5-year survival rate of NSCLC is still less than $15 \%$ due to the vast majority of patients that are diagnosed at an advanced stage with metastasis or relapse $(5,6)$. Tumor metastasis is a complex process involving multiple steps, including changes in tumor cell adhesion, matrix degradation, cell migration and invasion, angiogenesis and the ability of the immune system to combat invasion. (7-14). Lack of biomarkers for early diagnosis and metastasis markers are still some of the challenges in NSCLC. Therefore, identification of new functional genes and demonstration of their roles in NSCLC is essential for the development of specific diagnostic methods and the design of more effective therapeutic strategies for NSCLC patients (15). Placenta-specific protein 1 (PLAC1) protein is encoded by placenta-specific gene 1 , which is mainly localized in the cell membrane and involved in the regulation of trophoblast growth and differentiation. PLAC1 has been found to play an important role in placental and embryo development (particularly the brain development) (16-21). During embryo implantation, the invasion of trophocytes into the endometrium and the formation of blood vessels are very similar to the growth, invasion and migration of tumors (22). Recently, increasing evidence revealed that PLAC1 expression is activated in a variety of human cancers, including gastric, non-small cell lung, liver and colorectal cancer, primary colorectal adenocarcinoma, epithelial ovarian and breast cancer (16,23-30). In addition, increased expression of PLAC1 was found to be positively correlated with the degree of tumor invasion, lymph node metastasis and distant metastasis (31). However, the expression pattern and functional role of PLAC1 in NSCLC have not yet been well documented.

In the present study, we performed immunohistochemistry and qRT-PCR analysis of PLAC1 expression levels in NSCLC tumor and adjacent non-tumor tissues, and NSCLC cell lines. We found that PLAC1 expression was significantly upregulated in NSCLC tissues, and its increased level was associated with poor prognosis and shorter survival time. Applying loss of function analysis, we investigated the biological function of PLAC1 in NSCLC cells. Finally, mechanistic investigation was performed to determine its regulation of underlying targets and pathways in NSCLC cells. 


\section{Materials and methods}

Tissue collection. We obtained 88 NSCLC lung tissues from patients who underwent surgery at Jiangsu Province Hospital between 2012 and 2014 and were diagnosed with NSCLC based on histopathological evaluation. Clinicopathological characteristics, including tumor-node-metastasis (TNM) staging, were recorded. No local or systemic treatment was conducted in these patients before surgery. All collected tissue samples were immediately snap-frozen in liquid nitrogen and stored at $-80^{\circ} \mathrm{C}$ until required. The present study was approved by the Research Ethics Committee of Nanjing Medical University, China. Written informed consent was obtained from all patients.

Immunochemistry. Tumor core samples were transferred to blank wax block holes, with 3-5 independent sample points and $40-45$ points/chip array. Serial sections (5- $\mu \mathrm{m}$ thick) were deparaffinized and rehydrated with xylene, and a series of grades of alcohol. After epitope retrieval (it can re-expose the antigen epitope or amend the denaturing effects of chemical reagents and thermal applications); and inactivation of endogenous peroxidase, sections were blocked with $10 \%$ normal goat serum for $30 \mathrm{~min}$, and then sequentially incubated with a rabbit anti-PLAC1/CP1 antibody (R\&D Systems, Minneapolis, MN, USA) and horseradish peroxidase-conjugated anti-rabbit IgG [Cell Signaling Technology (CST), Danvers, MA, USA]. After being washed, the slides were developed using diaminobenzidine tetrahydrochloride and counterstained with hematoxylin.

Cell lines. Five NSCLC adenocarcinoma cell lines (PC-9, SPC-A1, H1299, A549 and H1650), 2 NSCLC squamous carcinomas cell lines (H520 and SK-MES-1) and human bronchial epithelial cells $16 \mathrm{HBE}$ were purchased from the Institute of Biochemistry and Cell Biology of the Chinese Academy of Sciences (Shanghai, China). A549, H1975, H1299, H1650 and H520 cells were cultured in RPMI-1640 medium; 16HBE, PC-9 and SPC-A1 cells were cultured in Dulbecco's modified Eagle's medium (DMEM) (Gibco-BRL, Grand Island, NY, USA) medium supplemented with $10 \%$ fetal bovine serum (FBS) $100 \mathrm{U} / \mathrm{ml}$ penicillin and $100 \mathrm{mg} / \mathrm{ml}$ streptomycin (Invitrogen, Carlsbad, CA, USA) at $37^{\circ} \mathrm{C} / 5 \% \mathrm{CO}_{2}$.

RNA extraction and qPCR assays. Total RNA was isolated using TRIzol reagent (Invitrogen) according to the manufacturer's instructions. Total RNA $(500 \mathrm{ng}$ ) was reverse-transcribed in a final volume of $10 \mu \mathrm{l}$ using random primers under standard conditions for the PrimeScript RT Reagent kit (Takara, Dalian, China). We used the SYBR Premix Ex Taq (TaKaRa) to determine PLAC1 expression levels, following the manufacturer's instructions. Results were normalized to the expression of $\beta$-actin (Invitrogen). The specific primers used were as follows: PLAC1 sense, 5'-CTGTCTTAGTCGCCTTCATGC-3' and antisense, 5'-TGAACCAATCTGTCGAGCACA-3'; $\beta$-actin sense, 5'-TCA CCC ACA CTG TGC CCA TCT ACG A-3' and antisense, 5'-CAG CGG AAC CGC TCA TTG CCA ATG G-3' (Invitrogen). The quantitative PCR (qPCR) assays were conducted on an ABI 7300 (Applied Biosystems, Foster City, CA, USA), and data was collected with this instrument. qPCR mainly included denaturation and annealing, the product length of which was expected to be between $80-150 \mathrm{bp}$. Our qPCR results were analyzed and expressed relative to threshold cycle (CT) values and then converted to fold changes.

Small interfering RNA duplexes and cell transfection. The si-PLAC1 (5'-GGGCACGCCAUCUAAGUUUTT-3') or si-NC (5'-UUCUCCGAACGUGUCACGUTT-3') (Invitrogen) were transfected into PC-9 and H1299 cells. PC-9 and H1299 cells were grown on 6-well plates to confluency and transfected using Lipofectamine 2000 (Invitrogen) according to the manufacturer's instructions. At $24 \mathrm{~h}$ post-transfection, the cells were harvested for qPCR or $48 \mathrm{~h}$ for western blot analysis.

Cell viability assays. Cell viability was monitored using a cell proliferation reagent kit I [Cell Counting Kit-8 (CCK-8)] (KeyGen Biotech, Shanghai, China). The PC-9 and H1299 cells transfected with si-PLAC1 (3,000 cells/well) were grown in 96-well plates. Cell viability was assessed every $24 \mathrm{~h}$ following the manufacturer's protocol. All experiments were performed in quadruplicate. For each treatment group, the wells were assessed in triplicate.

Flow cytometry. $\mathrm{H} 1299$ or PC-9 cells transfected with si-PLAC1 were harvested $48 \mathrm{~h}$ after transfection by trypsinization. After double staining with FITC-Annexin V and propidium iodide (PI) was carried out using the FITC-Annexin V apoptosis detection kit (BD Biosciences, San Jose, CA, USA) according to the manufacturer's recommendations, the cells were analyzed with a flow cytometer (FACScan) equipped with CellQuest software (both from BD Biosciences). Cells were discriminated into viable cells (Annexin V-negative and PI-negative), early apoptotic (Annexin V-positive and PI-negative), dead and apoptotic cells (Annexin V-positive and PI-positive), and then the relative ratio of early apoptotic cells was compared with the control transfectant from each experiment. Cells for cell cycle analysis were stained with PI using the Cycletest Plus DNA reagent kit (BD Biosciences) following the manufacturer's protocol and analyzed by FACScan. The percentage of the cells in the G0/G1, S and G2/M phases were counted and compared.

Cell migration and invasion assays. For the migration assays, at $48 \mathrm{~h}$ post-transfection, $5 \times 10^{4}$ cells in serum-free media were placed into the upper chamber of an insert $(8-\mu \mathrm{m}$ pore size; Millipore, Billerica, MA, USA). For the invasion assays, $1 \times 10^{5}$ cells in serum-free medium were placed into the upper chamber of an insert coated with Matrigel (Sigma-Aldrich, St. Louis, MO, USA). Medium containing 10\% FBS (Gibco-BRL) was added to the lower chamber. After incubation for $24 \mathrm{~h}$, the cells remaining on the upper membrane were removed with cotton wool. Cells that had migrated or invaded through the membrane were stained with methanol and $0.1 \%$ crystal violet, imaged and counted using an IX71 inverted microscope (Olympus, Tokyo, Japan). Experiments were independently repeated 3 times.

Western blot assay and antibodies. Cells protein lysates (Millipore) were separated by $10 \%$ sodium dodecyl sulfate-polyacrylamide gel electrophoresis (SDS-PAGE), transferred to $0.22 \mu \mathrm{m}$ NC membranes (Sigma, St. Louis, MO, USA), and incu- 
bated with specific antibodies. Enhanced chemiluminescence (ECL) chromogenic substrate (Beyotime, Shanghai, China) was used to visualize the bands and the intensity of the bands were quantified by densitometry (Quantity One Software; Bio-Rad, Hercules, CA, USA). $\beta$-actin antibody $(1: 5,000)$ was used as a control, and anti-PLAC1 (1:500) (ab117528), anti-vimentin $(1: 2,000)$ (AF2105), anti-AKT $(1: 5,000)$ (MAB2055), antipAKT (S473) (1:2,000) (AF887) and anti-E-cadherin (1:10,000) (AF648) were purchased from R\&D Systems. Donkey anti-goat IgG-HRP $(1: 5,000)$ (sc-2020) was purchased from Santa Cruz Biotechnology (Santa Cruz, CA, USA). Goat anti-mouse IgG-HRP $(1: 5,000)(\# 7076 s)$ and goat anti-rabbit IgG-HRP $(1: 5,000)(7074 \mathrm{~s})$ were purchased from CST.

Statistical analysis. All statistical analyses were performed using GraphPad Prism 5.01 software (GraphPad Software, Inc., La Jolla, CA, USA). The significance of differences between groups was estimated using the Student's t-test or $\chi^{2}$ test. Progression-free survival (PFS) rates were calculated using the Kaplan-Meier method with the log-rank test applied for comparison. Variables with $\mathrm{P}<0.05$ in univariate analysis were used in subsequent multivariate analysis on the basis of Cox regression analyses. Two-sided P-values were calculated, and a probability level of 0.05 was chosen for statistical significance.

\section{Results}

PLAC1 expression is upregulated in NSCLC and is correlated with poor prognosis. The expression and localization of PLAC1 protein was determined by IHC staining using anti-PLAC1 antibody. As shown in Fig. 1A, PLAC1-positive signaling was confined to the neoplastic cell population, but not in adjacent stromal and non-neoplastic epithelial cells. Moreover, the PLAC1 protein level was higher in the moderately- and poorly-differentiated tumor tissues than that in the highly-differentiated tumor tissues (Fig. 1B). In terms of clinical stage, the expression of PLAC1 in patients with advanced-stage tissue was higher than that in patients with early-stage tissue (Table I). Furthermore, the level of PLAC1 mRNA expression was determined in 88 NSCLC tissue samples by qRT-PCR. However, according to the histological classification, there was no significant difference in the expression of PLAC1 at the RNA level. To investigate the relationship between the expression level of PLAC1 and the clinical features of patients we classified these patients into 2 groups: the high-PLAC1 expression ( $n=44$, fold change $\geq$ mean ratio); and the low-PLAC1 expression groups $(n=44$, fold change $\leq$ mean ratio) (Fig. 1C). The association analysis revealed that higher PLAC1 expression was related with TNM stage $(\mathrm{P}=0.0335)$, and lymph node metastasis $(\mathrm{P}=0.0165)$ (Table I).

Furthermore, Kaplan-Meier survival analysis was conducted to investigate the correlation between PLAC1 expression and NSCLC patient prognosis. With respect to PFS, the median survival time for the low PLAC1 group was obviously far longer than that for the high PLAC1 group (Fig. 1D). The Kaplan-Meier survival curve revealed that 2 years of overall survival for low PLAC1 expression was $~ 95 \%$ while for the high PLAC1 expression it was 55\%. These findings support the hypothesis that PLAC1 overexpression plays an important role in NSCLC development and progression.
Table I. Correlation between PLAC1 expression and clinicopathological characteristics of NSCLC patients.

\begin{tabular}{|c|c|c|c|}
\hline \multirow[b]{2}{*}{$\begin{array}{l}\text { Clinicopathological } \\
\text { characteristics }\end{array}$} & \multicolumn{2}{|c|}{ PLAC1 } & \multirow[b]{2}{*}{$\begin{array}{c}\chi^{2} \text { test } \\
\text { (P-value }\end{array}$} \\
\hline & $\begin{array}{l}\text { Low, no. of } \\
\text { cases }(44)\end{array}$ & $\begin{array}{l}\text { High, no. of } \\
\text { cases (44) }\end{array}$ & \\
\hline Age, years & & & 0.3812 \\
\hline$\leq 65$ & 25 & 29 & \\
\hline$>65$ & 19 & 15 & \\
\hline Sex & & & 0.1697 \\
\hline Male & 27 & 33 & \\
\hline Female & 17 & 11 & \\
\hline Histologic subtype & & & 0.8131 \\
\hline $\mathrm{SCC}$ & 12 & 13 & \\
\hline Adenocarcinoma & 32 & 31 & \\
\hline TNM stage & & & $0.0335^{\mathrm{a}}$ \\
\hline $\mathrm{Ia}+\mathrm{Ib}$ & 25 & 17 & \\
\hline $\mathrm{IIa}+\mathrm{IIb}$ & 12 & 9 & \\
\hline III+IV & 7 & 18 & \\
\hline Tumor size $(\mathrm{cm})$ & & & $0.0314^{\mathrm{a}}$ \\
\hline$\leq 5$ & 30 & 20 & \\
\hline$>5$ & 14 & 24 & \\
\hline $\begin{array}{l}\text { Lymph node } \\
\text { metastasis }\end{array}$ & & & $0.0165^{\mathrm{a}}$ \\
\hline Negative & 32 & 20 & \\
\hline Positive & 12 & 24 & \\
\hline Smoking history & & & 0.6669 \\
\hline Smokers & 18 & 20 & \\
\hline Non smokers & 26 & 24 & \\
\hline
\end{tabular}

${ }^{\text {a }}<0.05$, overall; PLAC1, placenta-specific protein; NSCLC, non-small cell lung cancer; SCC, squamous cell carcinoma; TNM, tumor-node-metastasis.

Modulation of PLAC1 expression in NSCLC cells. We next performed qRT-PCR analysis to examine the expression of PLAC1 in 7 human NSCLC cell lines, including both adenocarcinoma and squamous carcinoma subtypes. The results revealed that PLAC1 expression was higher in the NSCLC cells than that in the normal cell line 16HBE. As H1299 and PC-9 had higher PLAC1 expression level, we chose these 2 cells lines for further investigation (Fig. 2A). Next, we detected the protein level of PLAC1 in H1299, PC-9 and SPC-A1 cells, and the results were consistent with the qRT-PCR results (Fig. 2B). To investigate the functional effects of PLAC1 in NSCLC cells, we knocked down its expression through transfection with PLAC1-specific siRNA duplexes, and a scrambled siRNA was used as a negative control. Then, qRT-PCR analysis determined that PLAC1 mRNA expression was decreased by $85 \%$ in $\mathrm{H} 1299$ cells and 75\% in PC-9 cells compared with the controls (Fig. 2C). Consistent with this observation, the PLAC1 protein was significantly decreased in both cell lines transfected with PLAC1 siRNA (Fig. 2D). 
A

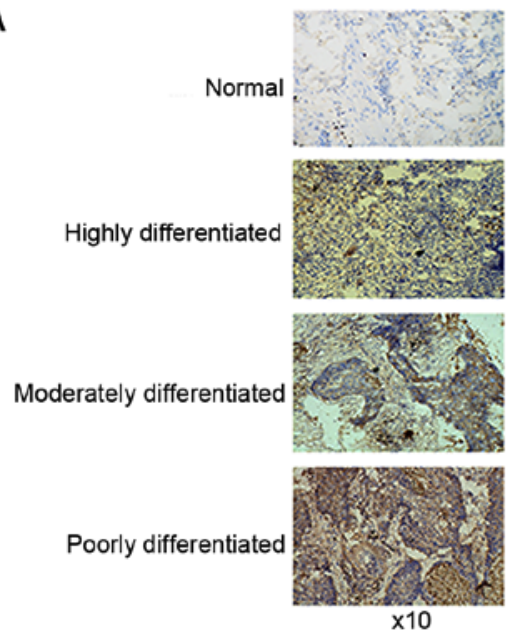

C

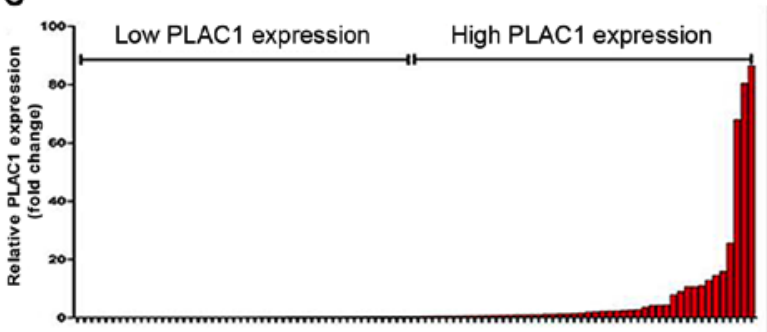

B
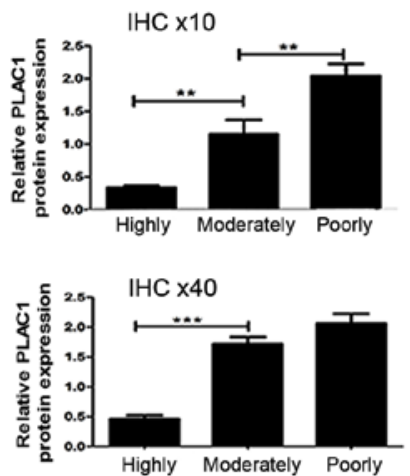

D

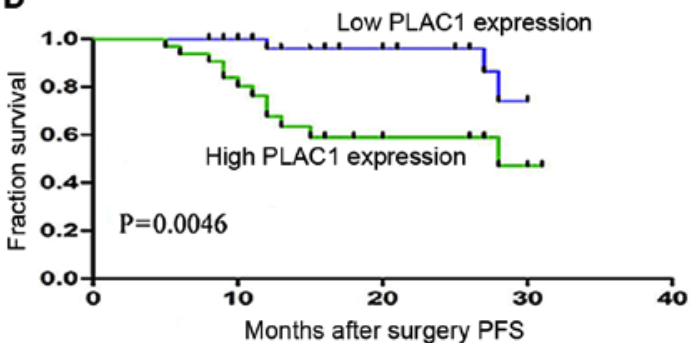

Figure 1. Relative PLAC1 expression in NSCLC tissues and its clinical significance. (A) The expression and localization of the PLAC1 protein in lung cancer tissue samples with different degrees of differentiation and normal tissue were determined by IHC staining using anti-PLAC1 antibodies as described in Materials and methods. Typical results are shown. (B) The level of PLAC1 was related with tumor stage. (C) Relative expression of PLAC1 in lung cancer tissues $(n=88)$ was examined by qPCR and normalized to $\beta$-actin expression. Results are presented as the fold change in tumor tissues and PLAC1 expression was classified into 2 groups. (D) Kaplan-Meier PFS curves according to PLAC1 expression levels; ${ }^{* *} \mathrm{P}<0.01,{ }^{* * *} \mathrm{P}<0.001$. PLAC1, placenta-specific protein 1; NSCLC, non-small cell lung cancer.

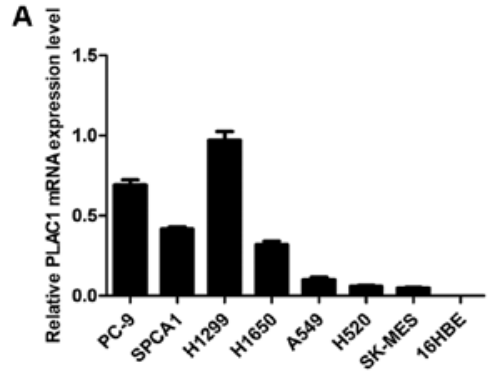

C

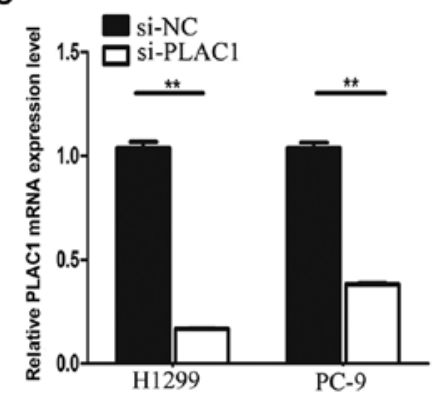

B

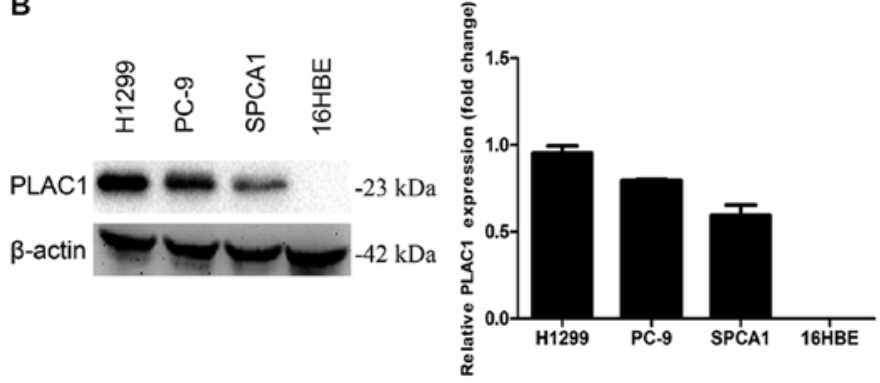

D

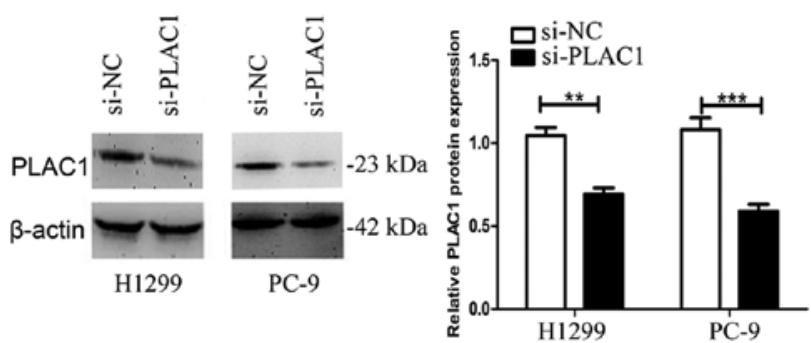

Figure 2. Effective knockdown of PLAC1 in NSCLC cells mediated by siRNA. (A and B) Results from qRT-PCR and western blotting demonstrating PLAC1 expression levels in NSCLC lines (PC-9, SPC-A1, H1299, A549, H1650, H520 and SK-MES-1) compared with a normal bronchial epithelium cell line (16HBE), (C and D) qRT-PCR and western blotting were performed to show effective knockdown of expression mediated by siRNA-PLAC1 in H1299 and PC-9 cells. PLAC1, placenta-specific protein 1; NSCLC, non-small cell lung cancer. 
A

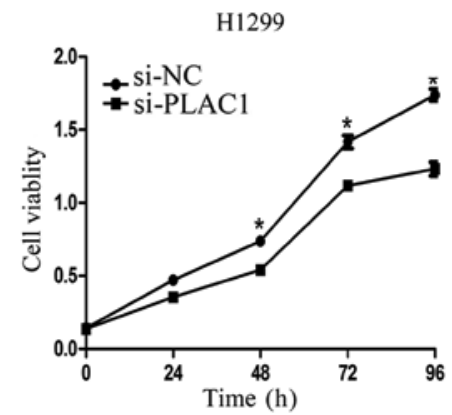

B
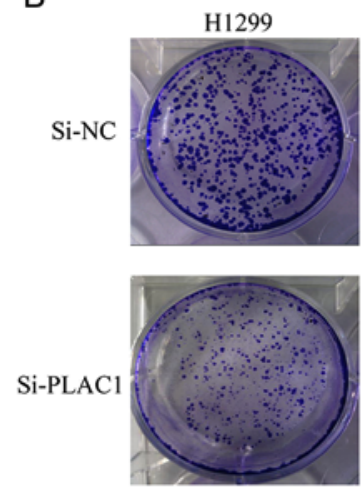
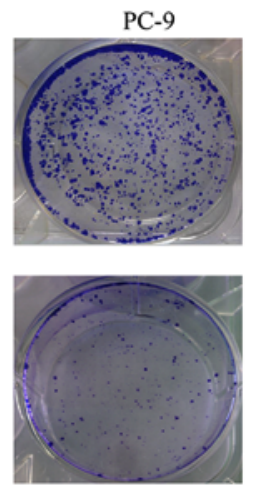
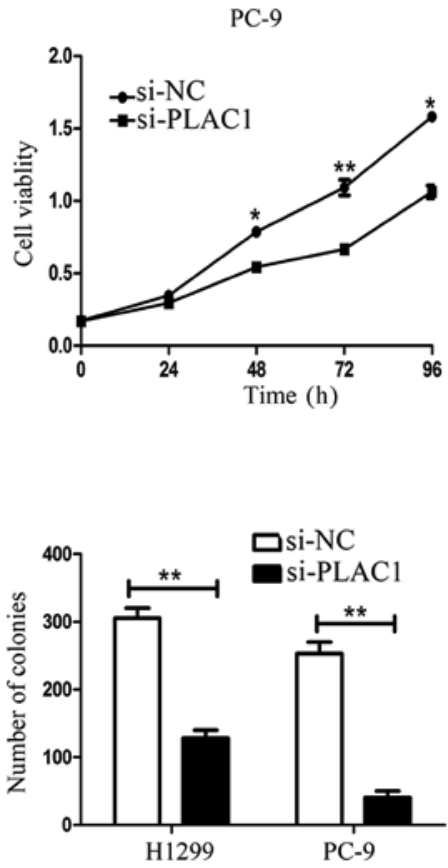

Figure 3. Effects of PLAC1 on NSCLC cell proliferation in vitro. (A) CCK-8 assays were used to determine the cell viability for si-PLAC1-transfected H1299 and PC-9 cells. Values represent the mean \pm SD, from 3 independent experiments. (B) Colony-formation assays were conducted to determine the proliferation of si-PLAC1-transfected H1299 and PC-9 cells. All experiments were performed in biological triplicates with 3 technical replicates; ${ }^{*} \mathrm{P}<0.05$, ${ }^{* *} \mathrm{P}<0.01$. PLAC1, placenta-specific protein 1; NSCLC, non-small cell lung cancer.

was inhibited in H1299 and PC-9 cells transiently transfected with si-PLAC1 compared with the controls (Fig. 3A). Colony formation assay results revealed that clonogenic survival was inhibited following downregulation of PLAC1 in H1299 and PC-9 cells (Fig. 3B).

To further examine the effect of PLAC1 knockdown on the cell cycle in NSCLC cells, cell cycle progression was analyzed by flow cytometry. The results revealed that $\mathrm{H} 1299$ and PC-9 cells transfected with si-PLAC1 had a marked cell cycle arrest at the G1/G0 phase and a decreased G2/S phase (Fig. 4A and B). To determine whether NSCLC cell proliferation was influenced by cell apoptosis, we performed flow cytometry. The results revealed that NSCLC cells transfected with PLAC1 siRNA exhibited a significant effect on apoptosis in PC-9 cells in comparison with that in the control cells, but not in the H1299 cells (Fig. 4C and D). Furthermore, in the H1299 cells a $\mathrm{G} 2 / \mathrm{M}$ arrest was noted in the si-PLAC1 group, but the opposite phenomenon was observed in PC-9 cells due to the difference in apoptosis between the 2 types of cells. Specifically, since the apoptosis in the si-PLAC1 group of PC-9 cells was significantly increased than that in the si-NC group, thus the total number of active cells in the cell cycle in the si-PLAC1 group was relatively less than that in the si-NC group, leading to the number of PC-9 cells in the G2/M phase to be relatively less in the si-PLAC1 group.

Decreased PLACl expression inhibits NSCLC cell migration and invasion. Cancer cell migration and invasion is a significant aspect of tumor progression. To investigate the effect of PLAC1 on NSCLC cell migration and invasion, we performed Transwell assays. After knockdown of PLAC1 in H1299 and PC-9 cells, we observed a marked reduction of the migratory capacity of these cells compared with the control cells (Fig. 5A). Moreover, invasive activity of H1299 and PC-9 cells was also inhibited by PLAC1 siRNA treatment (Fig. 5B). The results indicated that PLAC1 plays an important role in cancer cell migration, invasion and tumor progression.

PLACl is involved in the regulation of the AKT pathway and EMT in NSCLC cells. To explore the molecular mechanisms by which PLAC1 contributes to the phenotypes of NSCLC cells, we investigated potential targets involved in cancer cell proliferation, invasion and metastasis. The AKT pathway is an important regulator of cancer cell motility and migration (32-34). Thus this prompted us to analyze whether PLAC1 had an effect on the regulation of AKT kinase in H1299 and PC-9 cells. Western blot analysis revealed that silencing of PLAC1 led to a marked reduction of phosphorylated AKT levels in H1299 and PC-9 cells, suggesting that AKT kinase activation was involved in the execution of downstream effects of PLAC1 (Fig. 6A and B). In addition, previous studies indicated that epithelial-mesenchymal transition (EMT) was closely related to tumor migration and invasion. EMT is an evolutionarily conserved developmental process, which is induced during cancer progression and contributes to metastatic colonization. EMT endows metastatic properties in cancer cells by enhancing mobility, invasion and resistance to apoptotic stimuli (35-38). To assess the effects of PLAC1 on EMT-related protein expression such as E-cadherin and vimentin, siRNA specifically targeting PLAC1 was transfected into H1299 and PC-9 cells. As shown in Fig. 6C and D, the expression of E-cadherin was increased in PLAC1-knockdown H1299 and PC-9 cells. Additionally, the expression of vimentin was decreased in PLAC1-knockdown cells. These data 
A
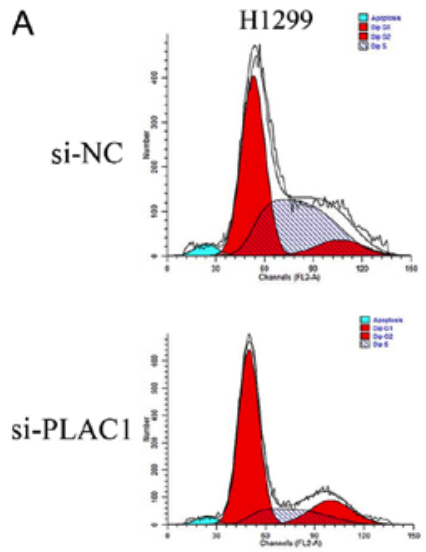

C

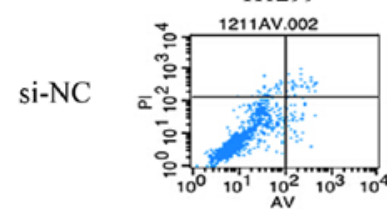

si-PLACl

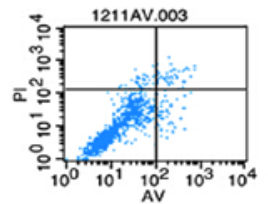

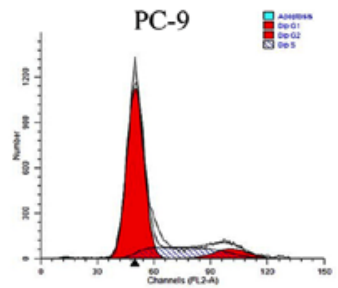

F

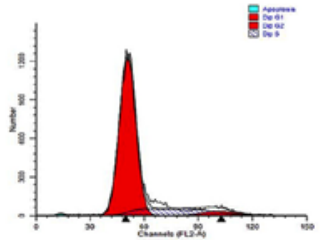

PC-9
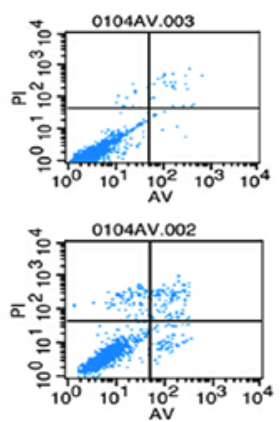

B

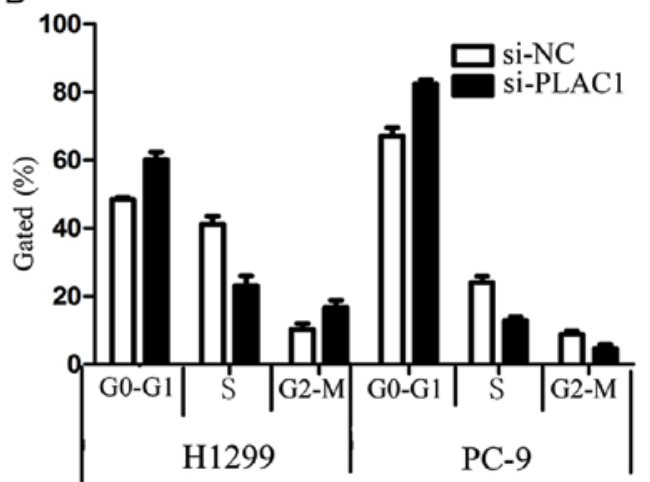

D

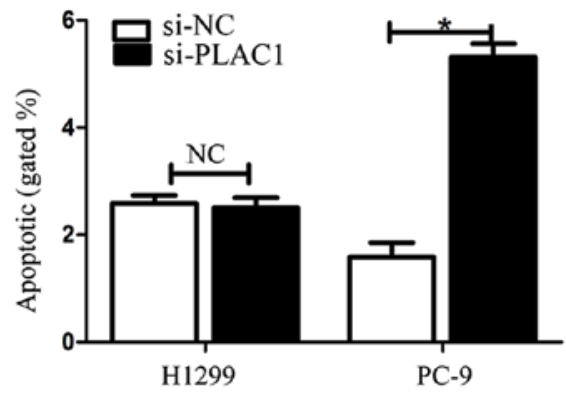

Figure 4. Knockdown of PLAC1 affects cell cycle progression and induces cell apoptosis in NSCLC cells in vitro. (A and B) Flow cytometric assays were performed to analyze cell cycle progression when NSCLC cells were transfected with si-PLAC1. The bar chart represents the percentage of cells in the G0/G1, S or G2/M phase, as indicated. (C and D) Flow cytometric assays were performed to analyze cell apoptosis in si-PLAC1-transfected H1299 and PC-9 cells. UL, necrotic cells; UR, terminal apoptotic cells; LR, early apoptotic cells. All experiments were performed in biological triplicates with 3 technical replicates; ${ }^{*} \mathrm{P}<0.05$. PLAC1, placenta-specific protein 1; NSCLC, non-small cell lung cancer.

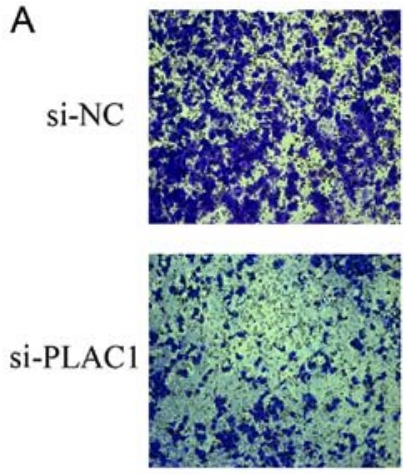

H1299

B

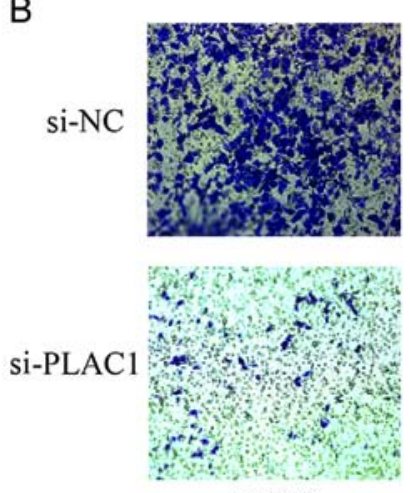

H1299
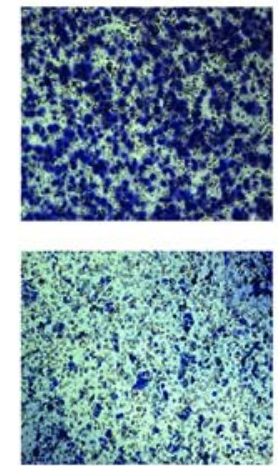

PC-9
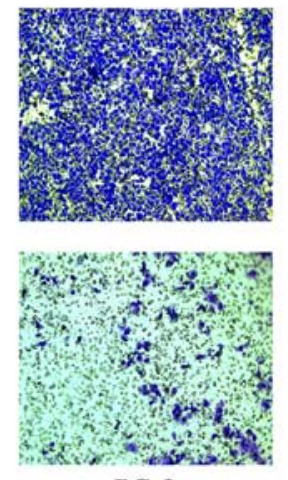

PC-9
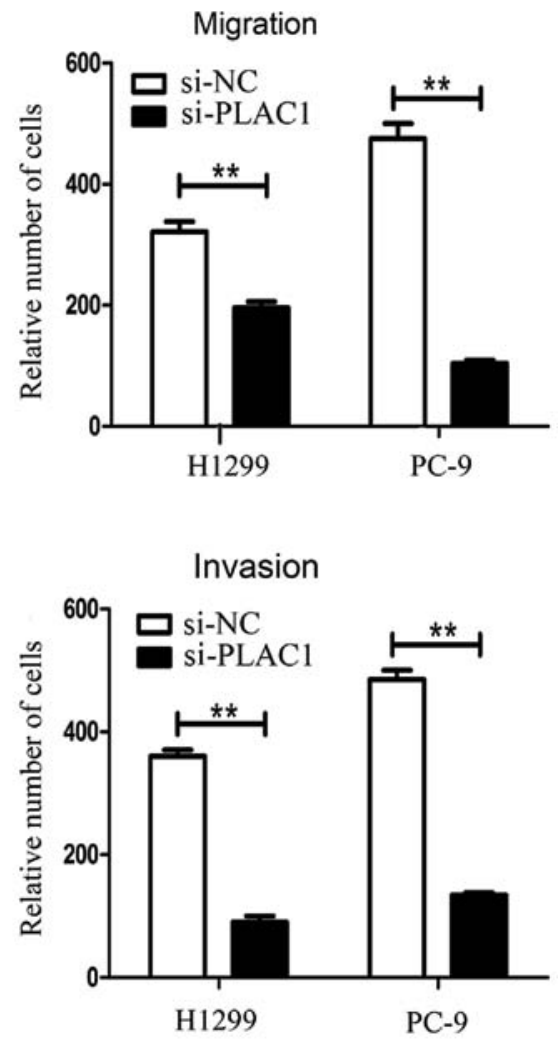

Figure 5. Effects of PLAC1 knockdown on NSCLC cell migration and invasion in vitro. (A) Effects of decreased PLAC1 on cell migration in si-PLAC1-transfected H1299 and PC-9 cells. (B) Effects of PLAC1 knockdown on cell invasion for si-PLAC1-transfected H1299 and PC-9 cells. Data are presented as the mean $\pm \mathrm{SD} ;{ }^{*} \mathrm{P}<0.05,{ }^{* *} \mathrm{P}<0.01$. PLAC1, placenta-specific protein 1; NSCLC, non-small cell lung cancer. 
A
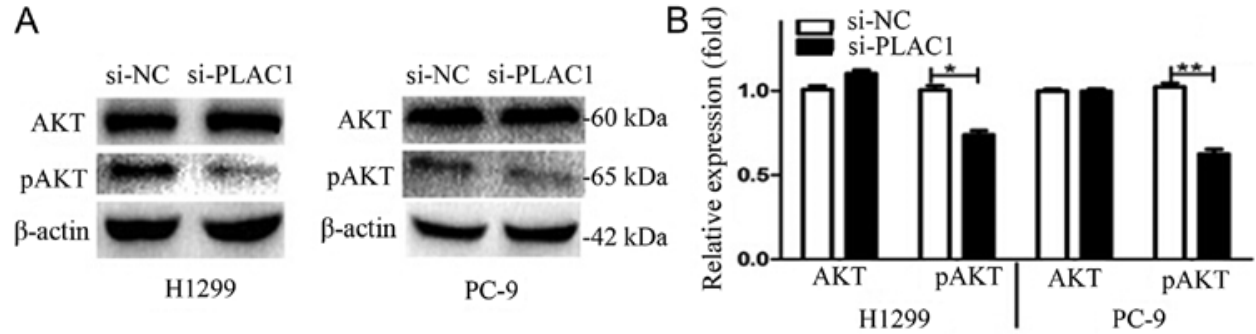

C
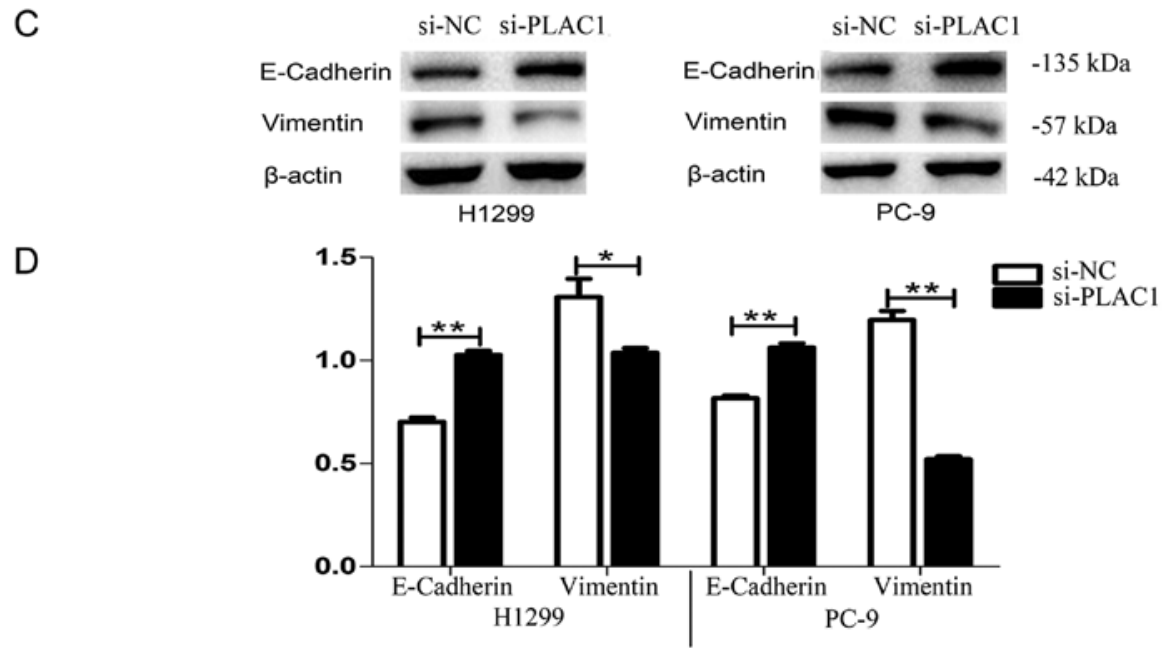

Figure 6. PLAC1 affects the AKT process and EMT-related protein levels. (A and B) AKT and pAKT proteins were detected in PLAC1-knockdown H1299 or PC-9 cells by western blotting. (C and D) The expression levels of E-cadherin and vimentin proteins were determined by western blotting in PLAC1knockdown H1299 and PC-9 cells. $\beta$-actin was used as an internal control. ${ }^{* *} \mathrm{P}<0.01$. PLAC1, placenta-specific protein 1; NSCLC, non-small cell lung cancer.

indicated that PLAC1 may influence the proliferation, invasive ability of NSCLC cells partly by altering the AKT pathway and EMT process.

\section{Discussion}

Effective control of cell proliferation and invasion is critical to the prevention of oncogenesis and successful cancer therapy (7). Therefore, identification of NSCLC-associated genes and investigation of their clinical significance and functions may provide a missing piece of the well-known oncogenic and tumor-suppressor network puzzle (15). In the present study, we evaluated RNA and protein expression patterns of PLAC1 in NSCLC cell lines and primary tumor samples from NSCLC patients. We found that the expression of PLAC1 was upregulated in NSCLC tissues and cells compared to normal tissues and cells. Moreover, an increased PLAC1 expression level was related to cancer cell differentiation and short survival time of patients. These findings indicated that PLAC1 played a direct role in the modulation of NSCLC progression and may be considered as a novel prognostic marker for NSCLC.

PLAC1 is a new tumor-associated gene, which was originally described as an X-linked gene $(39,40)$. PLAC1 expression is restricted primarily to cells derived from the trophoblast lineage, and is expressed during embryonic development (18). Several studies have revealed that PLAC1 expression in normal placenta and cancer cells is driven by specific interactions involving a combination of transcription factors. Recently, a number of studies have demonstrated the role of PLAC1 in placental development, pregnancy and host immune response against malignant tumors $(16,23)$. Notably, PLAC1 upregulation contributed to a range of biological functions and provided a cellular growth advantage, resulting in progressive and uncontrolled tumor growth.

To further investigate the biological role of PLAC1 in NSCLC, we explored the effects of PLAC1 on various aspects of NSCLC cell phenotype. RNAi-mediated suppression of PLAC1 in H1299 and PC-9 cells led to a significant inhibition of proliferation, migration and invasion, and cell growth arrest. To further document the molecular mechanism by which PLAC1 contributes to the NSCLC cell function, we investigated the potential target proteins involved in cell proliferation and invasion. In the present study, loss of PLAC1 in NSCLC cells led to a significant increase in the epithelial-mesenchymal transition-related protein levels of E-cadherin, and a decrease in vimentin. In addition, PLAC1 downregulation also reduced pAKT protein levels. Our findings indicated that PLAC1 contributed to NSCLC cell proliferation, migration and invasion possibly and partly via regulation of epithelial-mesenchymal transition and the AKT pathway.

In summary, the present study revealed that PLAC1 expression was highly expressed in NSCLC tissues, suggesting that PLAC1 may be a prognostic factor and higher risk for NSCLC patients. PLAC1 was involved in regulation of the proliferation, migration, and invasion abilities of NSCLC cells partly through regulation of epithelial-mesenchymal transition and the AKT pathway. These findings elucidated NSCLC pathogenesis and progression, and facilitate the development of tumor-associated gene-directed diagnostics and therapeutics against this deadly disease. 


\section{Acknowledgements}

The present study was supported by the National Natural Scientific Foundation of China (no. 81372397).

\section{References}

1. Siegel RL, Miller KD and Jemal A: Cancer statistics, 2015. CA Cancer J Clin 65: 5-29, 2015.

2. Travis WD, Brambilla E, Nicholson AG, Yatabe Y, Austin JH, Beasley MB, Chirieac LR, Dacic S, Duhig E, Flieder DB, et al; WHO Panel: The 2015 World Health Organization Classification of Lung Tumors: Impact of Genetic, Clinical and Radiologic Advances Since the 2004 Classification. J Thorac Oncol 10 : 1243-1260, 2015.

3. Herbst RS, Heymach JV and Lippman SM: Lung cancer. N Engl J Med 359: 1367-1380, 2008.

4. Chen W, Zheng R, Baade PD, Zhang S, Zeng H, Bray F, Jemal A, Yu XQ and He J: Cancer statistics in China, 2015. CA Cancer J Clin 66: 115-132, 2016.

5. Jung KW, Park S, Kong HJ, Won YJ, Lee JY, Seo HG and Lee JS: Cancer statistics in Korea: Incidence, mortality, survival, and prevalence in 2009. Cancer Res Treat 44: 1-24, 2012.

6. Chaari A, Ben Nasr S, Labidi S, Afrit M and Boussen $\mathrm{H}$ : Metastatic non-small cell lung cancer: A Tunisian retrospective study about 100 cases. Tunis Med 93: 294-296, 2015 (In French).

7. Geiger TR and Peeper DS: Metastasis mechanisms. Biochim Biophys Acta 1796: 293-308, 2009.

8. Chan DA and Giaccia AJ: Hypoxia, gene expression, and metastasis. Cancer Metastasis Rev 26: 333-339, 2007.

9. Headley MB, Bins A, Nip A, Roberts EW, Looney MR, Gerard A, and Krummel MF: Visualization of immediate immune responses to pioneer metastatic cells in the lung. Nature 531: 513-517, 2016

10. Rankin EB and Giaccia AJ: Hypoxic control of metastasis Science 352: 175-180, 2016.

11. Turajlic $\mathrm{S}$ and Swanton C: Metastasis as an evolutionary process. Science 352: 169-175, 2016.

12. Cheung KJ and Ewald AJ: A collective route to metastasis: Seeding by tumor cell clusters. Science 352: 167-169, 2016.

13. Kiberstis PA: Metastasis: An evolving story. Science 352: 162-163, 2016.

14. Tüting T and de Visser KE: CANCER. How neutrophils promote metastasis. Science 352: 145-146, 2016.

15. Swanton C and Govindan R: Clinical implications of genomic discoveries in lung cancer. N Engl J Med 374: 1864-1873, 2016.

16. Wang X, Baddoo MC and Yin Q: The placental specific gene, PLAC1, is induced by the Epstein-Barr virus and is expressed in human tumor cells. Virol J 11: 107, 2014.

17. Silva WA Jr, Gnjatic S, Ritter E, Chua R, Cohen T, Hsu M, Jungbluth AA, Altorki NK, Chen YT, Old LJ, et al: PLAC1, a trophoblast-specific cell surface protein, is expressed in a range of human tumors and elicits spontaneous antibody responses. Cancer Immun 7: 18, 2007.

18. Fant M, Barerra-Saldana H, Dubinsky W, Poindexter B and Bick R: The PLAC1 protein localizes to membranous compartments in the apical region of the syncytiotrophoblast. Mol Reprod Dev 74: 922-929, 2007.

19. Massabbal E, Parveen S, Weisoly DL, Nelson DM, Smith SD and Fant M: PLACI expression increases during trophoblast differentiation: Evidence for regulatory interactions with the fibroblast growth factor-7 (FGF-7) axis. Mol Reprod Dev 71: 299-304, 2005

20. Rawn SM and Cross JC: The evolution, regulation, and function of placenta-specific genes. Annu Rev Cell Dev Biol 24: 159-181, 2008.

21. Fant M, Weisoly DL, Cocchia M, Huber R, Khan S, Lunt T and Schlessinger D: PLAC1, a trophoblast-specific gene, is expressed throughout pregnancy in the human placenta and modulated by keratinocyte growth factor. Mol Reprod Dev 63: 430-436, 2002.

22. Chang WL, Yang Q, Zhang H, Lin HY, Zhou Z, Lu X, Zhu C, Xue LQ and Wang H: Role of placenta-specific protein 1 in trophoblast invasion and migration. Reproduction 148: 343-352, 2014.
23. Dong XY, Peng JR, Ye YJ, Chen HS, Zhang LJ, Pang XW, Li Y, Zhang Y, Wang S, Fant ME, et al: Placl is a tumor-specific antigen capable of eliciting spontaneous antibody responses in human cancer patients. Int J Cancer 122: 2038-2043, 2008.

24. Liu F, Shen D, Kang X, Zhang C and Song Q: New tumour antigen PLAC1/CP1, a potentially useful prognostic marker and immunotherapy target for gastric adenocarcinoma. J Clin Pathol 68: 913-916, 2015.

25. Liu F, Zhang H, Shen D, Wang S, Ye Y, Chen H, Pang X, Song Q and He P: Identification of two new HLA-A*0201-restricted cytotoxic $\mathrm{T}$ lymphocyte epitopes from colorectal carcinomaassociated antigen PLAC1/CP1. J Gastroenterol 49: 419-426, 2014.

26. Liu FF, Shen DH, Wang S, Ye YJ and Song QJ: Expression of PLAC1/CP1 genes in primary colorectal carcinoma and its clinical significance. Zhonghua Bing Li Xue Za Zhi 39: 810-813, 2010 (In Chinese).

27. Ghods R, Ghahremani MH, Madjd Z, Asgari M, Abolhasani M, Tavasoli S, Mahmoudi AR, Darzi M, Pasalar P, Jeddi-Tehrani M, et al: High placenta-specific 1/low prostate-specific antigen expression pattern in high-grade prostate adenocarcinoma. Cancer Immunol Immunother 63: 1319-1327, 2014.

28. Liu W, Zhai M, Wu Z, Qi Y, Wu Y, Dai C, Sun M, Li L and Gao Y: Identification of a novel HLA-A2-restricted cytotoxic T lymphocyte epitope from cancer-testis antigen PLAC1 in breast cancer. Amino Acids 42: 2257-2265, 2012.

29. Tchabo NE, Mhawech-Fauceglia P, Caballero OL, Villella J, Beck AF, Miliotto AJ, Liao J, Andrews C, Lele S, Old LJ, et al: Expression and serum immunoreactivity of developmentally restricted differentiation antigens in epithelial ovarian cancer. Cancer Immun 9: 6, 2009.

30. Koslowski M, Türeci O, Biesterfeld S, Seitz G, Huber C and Sahin U: Selective activation of trophoblast-specific PLAC1 in breast cancer by CCAAT/enhancer-binding protein beta (C/EBPbeta) isoform 2. J Biol Chem 284: 28607-28615, 2009.

31. Koslowski M, Sahin U, Mitnacht-Kraus R, Seitz G, Huber C and Türeci O: A placenta-specific gene ectopically activated in many human cancers is essentially involved in malignant cell processes. Cancer Res 67: 9528-9534, 2007.

32. Cantley LC: The phosphoinositide 3-kinase pathway. Science 296: 1655-1657, 2002.

33. Luo J, Manning BD and Cantley LC: Targeting the PI3K-Akt pathway in human cancer: Rationale and promise. Cancer Cell 4: 257-262, 2003.

34. Yeh PS, Wang W, Chang YA, Lin CJ, Wang JJ and Chen RM: Honokiol induces autophagy of neuroblastoma cells through activating the PI3K/Akt/mTOR and endoplasmic reticular stress/ERK1/2 signaling pathways and suppressing cell migration. Cancer Lett 370: 66-77, 2016.

35. Chen X, Guan X, Zhang H, Xie X, Wang H, Long J, Cai T, Li S, Liu Z and Zhang Y: DAL-1 attenuates epithelial-to mesenchymal transition in lung cancer. J Exp Clin Cancer Res 34: 3, 2015.

36. Mittal V: Epithelial mesenchymal transition in aggressive lung cancers. Adv Exp Med Biol 890: 37-56, 2016.

37. Pattabiraman DR, Bierie B, Kober KI, Thiru P, Krall JA, Zill C, Reinhardt F, Tam WL and Weinberg RA: Activation of PKA leads to mesenchymal-to-epithelial transition and loss of tumorinitiating ability. Science 351: aad3680, 2016.

38. Seton-Rogers S: Epithelial-mesenchymal transition: Untangling EMT's functions. Nat Rev Cancer 16: 1, 2016

39. Cocchia M, Huber R, Pantano S, Chen EY, Ma P, Forabosco A, Ko MS, Schlessinger D: PLAC1, an Xq26 gene with placentaspecific expression. Genomics 68: 305-312, 2000.

40. Fant M, Farina A, Nagaraja R and Schlessinger D: PLAC1 (Placenta-specific 1): A novel, X-linked gene with roles in reproductive and cancer biology. Prenat Diagn 30: 497-502, 2010.

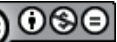

This work is licensed under a Creative Commons Attribution-NonCommercial-NoDerivatives 4.0 International (CC BY-NC-ND 4.0) License. 